\title{
The Research of Clothing Space Design
}

\author{
Qiao $\mathrm{CHEN}^{1}$ and Xiao-ping $\mathrm{HU}^{1,{ }^{*}}$ \\ ${ }^{1}$ School of Design, Guangzhou Higher Education Mega Centre, South China \\ University of Technology, Panyu District, Guangzhou 510006, People's Republic of \\ China
}

${ }^{*}$ Corresponding Author: huxp@scut.edu.cn

\begin{abstract}
Keywords: Architectural space design thinking, Clothing inside and outside space, Ergonomics, Modeling.
\end{abstract}

\begin{abstract}
This research mainly aims at the close relationship between clothing and human activities in people's daily life, trying to design different costumes to address the demand problem for clothing functionality that people usually have in different environment or different activities. It demonstrates that using ergonomics to discuss the issues related to anthropometric, anatomizing garment construction and other aspects so as to obtain the correct size plays an important role in designing different functional modeling clothing as well. In comparison of building space with clothing space, the author has been draw a conclusion that these two spaces have consistency, besides, it was discussed that East and West clothing have great contrasts in clothing dimensional modeling because of their different space. Based on that, this paper also describes the function of the costume inner space and outer space. In conclusion, the clothing space modeling is closely linked to ergonomics, people's exercise, apparel fabrics and so on.
\end{abstract}

\section{Introduction}

With the development of the social economy, modern science and technology are constantly making progress, people's living standards continue to improve, dressing requirement is no longer a simple warm but more to meet the demands of the individual spirit. In recent years, the concept of humane design has gradually penetrated into the design of costume space, it has become an inevitable trend in the clothing design that the object is the human-being, all the design is served for them and give them humane care. According to the psychological behavior of human-being, design that meet people's requirement of clothing comfort and make people have a good mood are catching more and more attention. The common of clothing and architectural space is both of them are designed for people and act for people, however, building space is a fixed visual style, while the clothing space is changed along with the movement of people, using three-dimensional thinking of architectural space to design a scientific and reasonable costumes, so as to meet the comfort of people dressed activities. According to the design relationship between the body and clothing space, using clothing ergonomic apply for the technical issues of the anthropometric measurements, anatomic clothing structure and other aspects. Therefore, the design could solve the problems of rationality, comfort, functionality, health and other issues, besides, it can meet the requirement of health, appearance and so on.

\section{The Concept and Commonness of the Space}

"Space", the term originated in the field of philosophy, refers to "the matter in motion 
exist extension and with objectivity" [1], with the expansion of the research in the field, it has extended out of the new and different meanings and applications in physics, mathematics, construction and other fields. In the 20th century, the construction is the largest sector which research and use the "space". The Interior of the architectural space is around its functions, the space functional can be divided into main house space, entertainment space, office space and so on. According to the type of human activities it can be divided into sports space and quiet space, due to different functions, the manner of processing is different. Whether it is indoor space, interior space, clothing space, they have a common characteristic is space. The space which human personal can be perceived in natural is three-dimensional space. A one-dimensional space is lines, dimensional space is flat, three-dimensional space is the length, width, height, a three-dimensional form. The characteristic of three-dimensional space is that it's encircled by the physical material. Various shapes such as rectangles, square, triangle, circle and so on which constitutes by different materials that people have seen in life. Because of different forms, disposing is different. As the proportion of building space will not only give people a psychological burden, but also bring inconvenience in people's life when it's not in line with the law of life, when the ratio of the building space is in line with people's habits, in which people will feel more comfortable instead. However, it is a conscious use of the influence of spatial scale on human psychological behavior to achieve the desired spatial effect in the design of architectural space. One example is that the oversized luxury space in five-star hotel will make people unconsciously awe of superior elite, another example is the daily living spaces such as dining room, living room, bedroom and so on can create a warm, comfortable space if they're designed according to the proportion of the ergonomically space. However, in the clothing, it refers to the empty space between the part of the body and the clothing, the clothing and the clothing in the condition of human body is dressing. The human body and clothing constitute the dressing space together, they have a direct impact on the costumes, fit, comfort, etc. Shaping of costumes is based on the clothing fit and comfort, the purpose of costume design is human-centered. So the design needs to meet the functional and comfort requirements of the wearer. Clothing space and building space have some commonality, they are constructed with different materials, the purpose of the design is based on human-centered. The difference is that the building is fixed visual style, clothing space is changed along with the movement of people.

\section{The Concept of Clothing Space}

\section{The Outline of Ergonomics}

Ergonomics is a studying "man - machine - environment" subject, which is based on physiology and psychology and combined with relevant knowledge, its aim is to use the theory of human science, combine the methods and production technology, besides it's outcome is directly serve for the production and the real life [2]. That is to say by the way of clothing Ergonomics to studying the relationship between body and garment structure to propose theoretical and practical basis carrier. If so, we'll view how to meet the human body's needs as a starting point, then to design a fit, comfort, and best costume. Ergonomics is a comprehensive discipline, which includes psychology, anatomy, anthropometry, fashion design school, and so on. Human-being are in a dominant position, both the equipment and the environment are at the service, it's aim is "people-oriented, human services," which emphasizes through 
people-oriented to study people, clothing, shelter, and all production activities, harmonize people, matter and the environmental systems, along with researching and developing scientifically, rationally and high efficiency.

\section{The Approach of Clothing Space}

The shaping of costumes is to make use of the outside space, which beyond the human body, to create a three-dimensional image that constitute by the human body and fabrics together, with the fabric properties and process tools to meet the functional and aesthetic needs of the wearer's [3]. For the clothing modeling expression have different interpretations, the western three-dimensional design emphasized people-centered and self-expression. In the eighteenth-century, French court culture is the representative of European culture, especially the rococo-style clothing in the baroque period, which clothing space constitution is grander, and the cultural background that was embodied is very on behalf of the European clothing and model. It's popular in the baroque period that women wear "restraint" inside and wear chest gorgeous embroidery and ornaments "V-type chest decorations" dress pannier rack outside, which is called "barge basket pannier rack." (Fig. 1) [4] Large and more external layers of the dress, with the flouncing ruffle sleeve, constitutes an overlaps garment inside space. The upper of the dress is fitting well, therefore, it can prominent women's curvy shape and showing particular charm, the lower part is very exaggerated and has occupied a large space. (Fig. 2) It's plump shape reflects a luxury style, the style of modern wedding dress is evolved at that time. In the 18th century, in order to achieve the visual effect on the shape of the space, the Western use iron to produce bras, it can make the upper of the human body to reach "V" shape, it is very beautiful and spiritual. This front of this dress is flat, the back is flat as well, the racking panniers constitute a generous space in the hem. Therefore, different spatial modeling on the human body have different visual effects. Spatial modeling is closely related to ergonomics, human movement amount, fabrics. Similarly, the modern history of Western women's clothing is a process of spatial variation. From the S-type of the early 20th century, and H-type in 1920s to broad shoulders modeling in 1930s, etc. the designer of the costumes continued to expand, as well as a variety of costumes were wantonly deducted under the postmodernism. Above all, it demonstrates the three-dimensional clothing modeling is also a use of spatial concepts

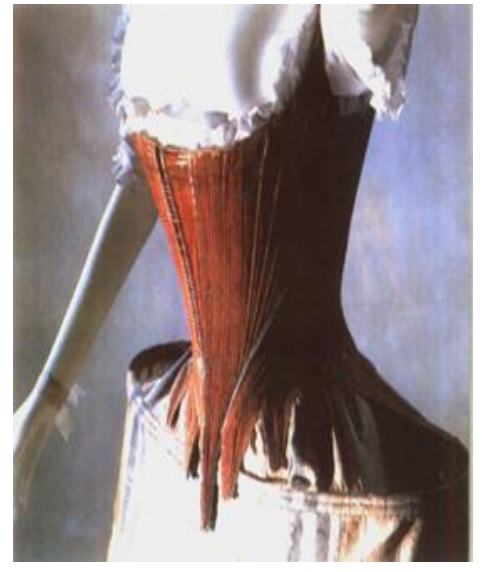

Figure 1: The Racking Panniers in 1775

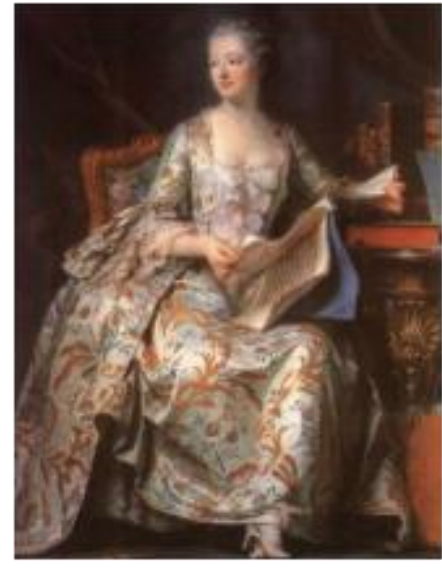

Figure 2: Women's Clothing in the 18th Century

The design of clothing spatial is rely on clothing structure, clothing structure is attached to ergonomics to measure, model the body and design the pattern's process parameters, through the relationship between body structure and methods of costume 
structural design to make clothing decent, comfortable and aesthetic. Finally it can make human, clothing and the environment much more harmony, provide reliable scientific evidence for the functional studying of the human body and the clothing and to achieve the concept that it can design starting point and fundamental purpose--"people-oriented, human services". Clothing style belongs to a space design configuration, the truth is costume derived from spatial structure and react to it. Thus, people with the actual situation space are interdependent and mutually constraining, not a simple garment itself. What's more should pay attention to is the interaction of space and clothing, if so it can constitute a unified whole of the "Time - Environment - Human Being".

\section{The Consistency of Clothing space and Building Space}

The common points of clothing and architecture is that the shape is built with different materials, whose design objects are people and the purpose is to serve people. Both of them are typical three-dimensional structure, so there exists space. However, the ultimate goal of architecture is to create a physical space that is in line with human needs, in clothing, space refers to the empty between clothing and human body, clothing and clothing with body dressed, the human body and clothing together constitute a dress space, which has a direct impact on the clothing shape, fitness, comfort and so on. The shape of clothing is based on the fitness and comfort of clothing. Therefore, not only should we meet the functional requirements of people who get dressed, but we should meet the comfort needs of them. They all have inner space and outer space, and people have different esthetic demand for garment modeling in different time, area and cultural background, thus it creates different spatial form the image of clothing.

Both clothing and architecture are typical three-dimensional structure, thus there exists space, and the ultimate goal of architecture is to create a physical space that is in line with human needs, both of them are people-oriented space creating. Interior space of the building is formed by surrounding entities like floor, walls and ceilings. Internal space has the closest relationship to human, and it has the greatest impact on human. Accordingly, the interior space of the garment refers to the space between the human body and the clothing, and the inner part of the clothing when people are in a static or moving time. In addition to protection, heating, cooling and other functions, the interior space of the garment has a significant impact on overall form of clothing constituted of three-dimensional modeling, including the basic space of human activities and the space required for three-dimensional modeling. In summary, it is no doubt that the inner space and outer space of construction and their relationship is consistent with the constitute of clothing space.

\section{The Classification and Function of Clothing Space}

\section{The Inner Space and Outer Space of Clothing}

Clothing is constituted by the three-dimensional modeling, there is the inner space and outer space environment, while the outer space environment of clothing is constituted by different permutations and combinations form of the inner space. Therefore, the spatial relationship between the internal apparel and the human body is called the inner of clothing, and the external apparel space is outside clothing space, which related to their own behavior and an external meaningful space, it has a great relationship with the people's living environment, working conditions and social environment. [8] The outer clothing space is constituted by overlapping, openness and 
image. The design outer clothing space not only provides a different shape image, but also makes the costumes more three-dimensional.

\section{The Function of Clothing Space}

Using padding, support, relaxation and other methods in design to make changes in the inner clothing space, so that to produce multi-style clothing in shape. One example is embedding elastic strip material to support fabric in the side seams and princess line, and using multilayer mesh organza to form the external swelling shape. Another example is the women's masculine T-shape, adding shoulder pads in the clothing shoulder to change the petite female body, the down with natural feather to pad the interior space, not only soft and warm, but breathable, feather filling clothing can be compressed within the space, so that clothing size is very small, and it's easy to save, etc. [6] therefore, the form of the inner clothing image is influenced by the structure, fabric and crafts of the clothing. The external clothing space is outside clothing space, which related to their own behavior and an external meaningful space, it has a great relationship with the people's living environment, working conditions and social environment, the outer clothing space is constituted by overlapping, openness and image, for example, creating various shapes of pockets and overlapping multilayer sleeves in the clothing not only reflect different space image, but also make the costumes more three-dimensional. From the perspective of shape of clothing, the outer clothing space can be showed by a variety of shapes and forms, it can be said the constitution of outer clothing space affect the overall image.

Therefore, the design of outer clothing space should pay full attention to research the clothing space imagery, and to obtain a more accurate prediction of spatial behavior, so that the designer can organize the various elements of outer clothing space.

\section{Perception Survey of Clothing Space Images}

\section{Researching Before Designing}

People are not only satisfied with the function of clothing that could provide protecting, keeping warm or cool, but also pursue higher value expression of dress requirement with economic development. Clothing plays an important role in our daily life. Because of the difference of location, race, culture and religion, people have different demanding of clothing form. Different environment and location correspond to different clothing external space. Social artifact adapts to the change of the external environment by adjusting internal system so that could achieve one's goal. Designing is a medium which could communicate with each other. Apparently many clothing factories would do some perception survey about clothing internal or external space before designing.

\section{Importance of Survey}

Firstly, we should focus on the age of target group, personality and place of correlation behavior to do the research before the stage of development and design. The scale of the survey contains the place of correlation behavior of target group such as making friends, working, shopping, relaxing and entertainment and we could observe the place mentioned above. The meaning of observation reflected in the compact degree between the place and the relation with our daily life [7]. What the foreground of designing need is life criterion of citizens which reflected the feeling of people of living in those places and it is the key factor to support a design from 
understanding external factor to internal factor to go through further research. Only using reasonable research data as the important support point can we design the works which meet people's needs. Therefore, more and more costume designer considered both people's need and the harmony and tacit relationship among people, environment and garment, which changed the former fashion designing concept that focus on formal beauty to show the rationality and scientificity of the designing requirement. There are some modern fashion designing concepts, such as security, healthy, comfort, function, beauty and personality. All in all, we should do some research about the external factor as scientific and reasonable basis, though clothing internal factor's functions were equally important.

There are more and more consensuses about the expression of clothing space, which from aesthetic taste to clothing external expression. The expression of dressing concept also became much more brightly. There is a goal behind the personality psychology, which is the requirement to the garment. The goal needs to satisfy with people's psychological requirement which make them feel leisurely, carefree, comfortable and beautiful and function as much as the clothes can. Besides, it can also fulfill the people's life and elevate their sentiment.

\section{Conclusion}

Generally speaking, the so-called angle of space design thinking is a comprehensive, multi-angle, multi-level thinking. In fact, space thinking of clothing design is based on the concept of space and awareness, using three-dimensional mode to express the image which formed in the costume design thinking. If there is the concept of inner and outer space images in the designer's mind, and can realize that he should take the space actors feel in space design into consideration. For any costume design, It's much more clear for solving the problem, and it's where the costume could make a progress.

\section{References}

[1] Men delai, Hu Xiaoping. The new architecture Comprehensive university art and design disciplines basic teaching system [J]. South China University of Technology (Social Science Edition) Volume 13, Issue 2. April 2011.

[2] Zhan Heping. Space [M]. Southeast University Press, 2006.

[3] Pan Jianhua. Clothing ergonomic [J]. Donghua University Press, 2008.

[4] Xu Ke, Liu Xiaogang. Costume design [M]. Donghua University Press, 2011.

[5] Ye Licheng. Western clothing history [M], China Textile Press, 2012.01.

[6] Pan Jianhua. Clothing ergonomics and design [M] Shanghai: Donghua University Press, 2008.

[7] Wang Xiaoyue. The inner space of clothing [M] Shanghai: Shanghai Science and Technology Press, $2004.78-83$.

[8] Guan Shaoping. Create outdoor space imagery [J]. Mechanical Engineering, South China University of Technology.

[9] Ma Lili. Design of costume space [J]. Progress in Textile Science and Technology, 2008. 